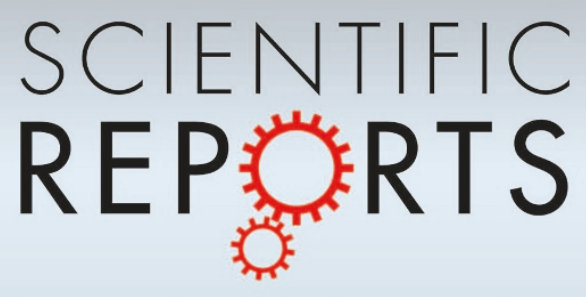

OPEN

SUBJECT AREAS:

AMOEBOID MIGRATION

COLLECTIVE CELL MIGRATION

CHEMOTAXIS

BIOLOGICAL PHYSICS

Received

22 May 2013

Accepted

3 July 2013

Published

29 July 2013

Correspondence and requests for materials should be addressed to H.K. (hidekuwayama@ biol.tsukuba.ac.jp)

\title{
Biological soliton in multicellular
} movement

\author{
Hidekazu Kuwayama' \& Shuji Ishida²
}

\begin{abstract}
'Faculty of Life and Environmental Sciences, University of Tsukuba, Tsukuba, Tennodai, 1-1-1, Ibaraki 305-8572, Japan,
${ }^{2}$ Department of Botany, Graduate School of Science, Kyoto University, Sakyo-ku, Kyoto 606-8502, Japan.
\end{abstract}

Solitons have been observed in various physical phenomena. Here, we show that the distinct characteristics of solitons are present in the mass cell movement of non-chemotactic mutants of the cellular slime mould Dictyostelium discoideum. During starvation, $D$. discoideum forms multicellular structures that differentiate into spore or stalk cells and, eventually, a fruiting body. Non-chemotactic mutant cells do not form multicellular structures; however, they do undergo mass cell movement in the form of a pulsatile soliton-like structure (SLS). We also found that SLS induction is mediated by adhesive cell-cell interactions. These observations provide novel insights into the mechanisms of biological solitons in multicellular movement.

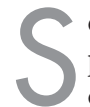

olitons are a widely observed physical phenomenon that behave like waves but possess many features of particles ${ }^{1}$. A soliton is defined as a self-reinforcing solitary wave that travels at constant speed without changing shape. Solitons do not obey the superposition principle, which makes the wave structure robust in collisions with other wave structures. In biology, soliton theory has been applied to explain signal and energy propagation in biomembranes, the nervous system, and low frequency collective motion in proteins and $\mathrm{DNA}^{2-5}$; however, there has been no evidence of solitons as a higher-level biological phenomena. An example of such biological phenomena is multicellular movement during morphogenesis and development. The details of how this organized phenomenon is spatiotemporally regulated have not been reported, but presumably such regulation is characterized by simple and robust rules.

We have demonstrated that non-chemotactic Dictyostelium discoideum mutants exhibit a characteristic structure with the features of a self-reinforcing solitary wave, or soliton. Under starvation conditions, the mutants do not aggregate but form an arc-shape multicellular structure, named the Soliton-Like-Structure (SLS). SLS movement continues much longer than the developmental cycle, moves at constant speed without changing shape, and does not obey the superposition principle. Even after collisions, waves pass through each other, conserving their physical qualities. Thus, we conclude the SLS exhibits soliton features that may be maintained by cell-to-cell adhesion mechanisms.

\section{Results}

Non-chemotactic mutants of Dictyostelium discoideum exhibit a soliton-like structure in multicellular movement. In wild-type cells of the slime mould Dictyostelium discoideum, a starvation signal triggers a developmental programme. Following exhaustion of their bacterial food source, the erstwhile random movement of the amoeboid cells is orchestrated to yield an aggregation of cells, mediated by the self-secreted extracellular chemoattractant, cAMP. The resultant migratory, multicellular structure is known as a slug. Transformation of the slug occurs within $8 \mathrm{~h}$ and results in a fruiting body that consists of two differentiated cell types, spore cells and stalk cells ${ }^{6}$ (Fig. 1a, Supplementary video S1).

We have isolated $D$. discoideum mutants that lack all chemotactic activities, leaving them unable to proceed down developmental pathways that require cell aggregation ${ }^{7.8}$. Soliton-like structures (SLSs) were observed in the KI-5 and KI-10 mutants within $6 \mathrm{~h}$ following the consumption of bacteria (Fig. 1b, Supplementary video S2). Since the features of the SLSs were not distinguishable between the two mutants, we only describe the KI-5 phenotype in this manuscript. SLSs emerged around $6 \mathrm{~h}$ after exhaustion of the bacterial food supply and persisted for $48 \pm 3 \mathrm{~h}$ in 5 independent experiments. (See a typical time course of SLS in Supplementary Fig. S1 and Supplementary video S3). Maintenance of the SLS structure despite collision with other SLSs is characteristic of a soliton wave (Fig. 1c, Supplementary video S4). The formation of SLSs is dependent on cell density such that the higher the density of the cells, the larger the SLS formed (Fig. 1d); however, an excessively high 
a

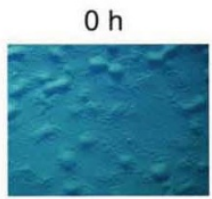

$16 \mathrm{~h}$

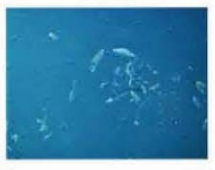

C

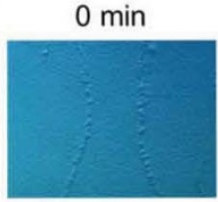

90 min

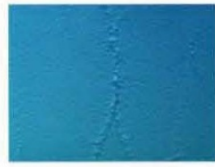

$135 \mathrm{~min}$

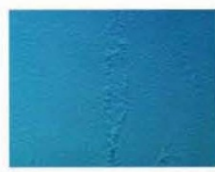

XP55

$8 \mathrm{~h}$

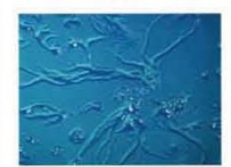

$20 \mathrm{~h}$

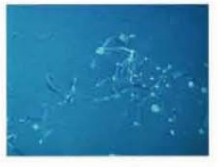

$60 \mathrm{~min}$

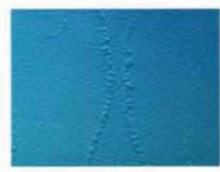

$105 \mathrm{~min}$

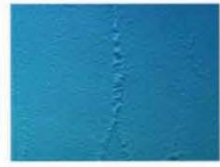

$150 \mathrm{~min}$

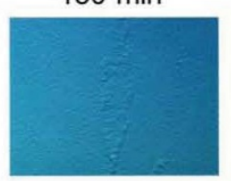

$12 \mathrm{~h}$

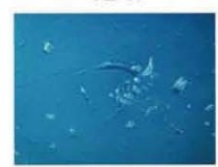

$24 \mathrm{~h}$

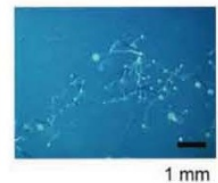

$1 \mathrm{~mm}$

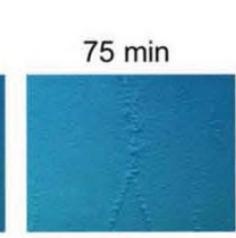

$120 \mathrm{~min}$

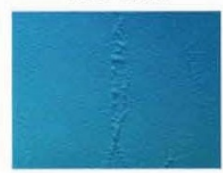

$180 \mathrm{~min}$

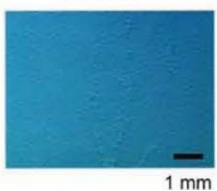

b

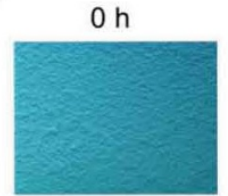

$7 \mathrm{~h}$

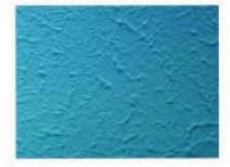

$8 \mathrm{~h}$

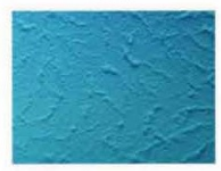

d

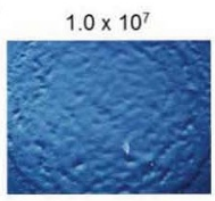

$2.0 \times 10^{5}$

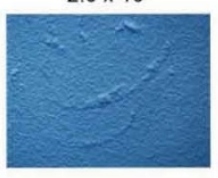

$1.0 \times 10^{5}$
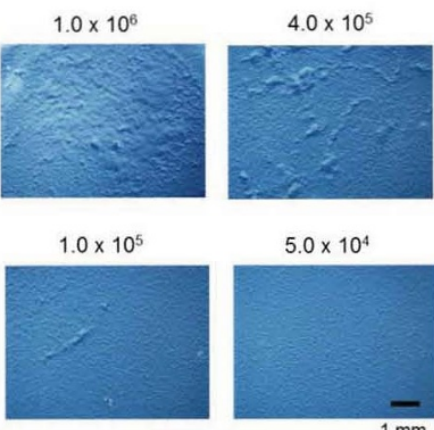

$5.0 \times 10^{4}$

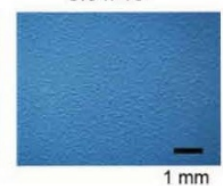

Cell density $=$ cells $/ \mathrm{cm}^{2}$

Figure $1 \mid$ Multicellular movement of the non-chemotactic $D$. discoideum KI-5 mutant shows soliton-like structures (SLSs) which behave similarly to soliton waves. (a, b), Formation of multicellular structures in parental wild-type XP55 (a) and SLSs in non-chemotactic mutant KI-5 (b) cells.

(c), The collision of 2 independent SLSs. (d), Dose dependency of KI-5 cell density on SLS formation. All pictures were taken at the indicated time after exhaustion of the D. discoideum food source, Klebsiella aerogenes.

density prevents SLS formation (Fig. 1d). Moreover, as the duration of cell starvation increased, SLS size and number decreased (Supplementary Fig. S1, Supplementary video S3). Despite the variability in size, SLSs moved at a constant velocity of approximately $20 \mu \mathrm{m} / \mathrm{min}$, which is twice the rate $(10 \mu \mathrm{m} / \mathrm{min})$ of starved wild-type cells ${ }^{9,10}$. Since SLS velocity is comparable to that of prespore or prestalk cells in the slug ${ }^{11}$, SLS cell motility may represent a distinct aspect of differentiated cell movement.

SLS depends on the CAMP signalling genes but is independent of extracellular cAMP and DIF-1. In D. discoideum, the sensory signalling pathway used during development involves cAMP synthesis and signalling through the serpentine cell surface receptor, trimeric $\mathrm{G}$ protein ${ }^{12}$. Therefore, mutants that lack the genes responsible for these processes-carA; the cAMP receptor, gpaB; the trimeric G protein $\alpha$ subunit, and acaA; an adenylyl cyclase-are defective in cell aggregation and dependent processes due to lack of cAMP production $^{13-15}$. These null mutants showed few signs of SLS formation, indicating that the cAMP signalling pathway is required for SLS formation (Fig. 2a, Supplementary video S5, S6, S7). We have shown that KI-5 and KI-10 are defective in cGMP metabolism in response to extracellular cAMP stimuli despite possessing the machinery for cAMP receptor-dependent cAMP production ${ }^{7,16,17}$. During cell aggregation, extracellular cAMP concentrations of an order of magnitude less than the micromolar level play a critical role such that cAMP concentrations of a millimolar order of magnitude substantially inhibit morphogenesis ${ }^{18}$. Next, in order to determine if extracellular cAMP is required for SLS formation, KI-5 cells were exposed to extremely high concentration and depletion of cAMP. Under the condition with the high concentration of $1 \mathrm{mM}$ cAMP, formation of SLSs was not disturbed (Fig. 2b). Furthermore, KI-5 cells were plated on non-nutrient agar with $4 \mathrm{mM}$ caffeine and conditioned medium prepared from the supernatant of wild-type cells in starvation (see Methods). Since caffeine is an adenylyl cyclase inhibitor in $D$. discoideum and the conditioned medium contains enough phosphodiesterase activity to degrade most of the physiological amount of extracellular cAMP ${ }^{19,20}$, little cAMP was measured in the extracellular fraction of KI-5 cells starved for $12 \mathrm{~h}$ with $5 \mathrm{mM}$ caffeine and conditioned medium (Table I; see Supplementary methods). With $5 \mathrm{mM}$ caffeine and the conditioned medium, SLSs were normally formed without disturbance (Fig. 2c). These observations indicate that extracellular cAMP is not required for SLS formation. Another morphogen, DIF-1, is a stalk cellinducing factor and a modulator of chemotactic activity ${ }^{21,22}$. In the presence of high DIF-1 concentrations, SLSs developed normally (Fig. 2d).

SLS is a dynamically stabilized structure. Cell motion in SLSs was investigated at a higher magnification and with RFP (Red fluorescent protein)-expressing KI-5 cells generated by transformation with 
$12 \mathrm{~h}$
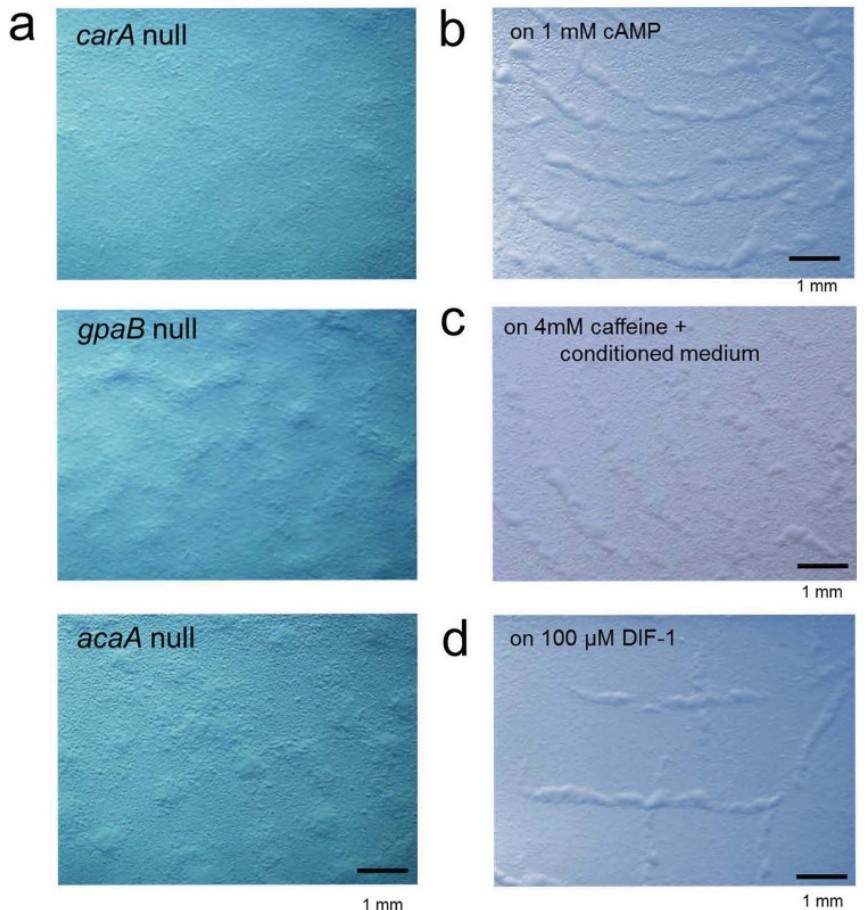

C

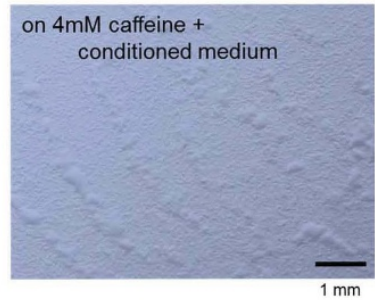

d

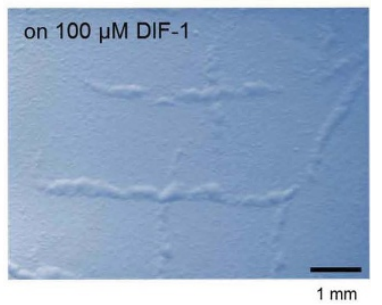

Figure $2 \mid$ Failure of SLS formation in non-chemotactic mutants with defective cAMP signalling, and the effect of CAMP and DIF-1 on SLS formation. (a), Absence of multicellular structure formation in carA, $g b p B$, and acaA null mutants following bacterial exhaustion. (b), SLS formation with $1 \mathrm{mM}$ cAMP. (c), Absence of SLS formation with $4 \mathrm{mM}$ caffeine. (d), SLS formation with $100 \mu \mathrm{M}$ DIF-1.

monomeric RFP expression vector. Motile cells in front of the wave moved randomly and did not appear to migrate into the SLS but instead were incorporated passively (Fig. 3a, 3b \& Supplementary video S8, S9, S10). At the same time, some cells at the rear of the SLS were left behind and reverted to random motion. The number of RFP-expressing incorporated cells was comparable to the number of cells left behind the SLS (Fig. 3b). In 3 independent SLSs, the number of highly RFP-expressing incorporated cells and the number of cells left behind were nearly the same (Table II). Furthermore, removal of cells in front of a moving SLS showed that the thickness of the SLS was reduced and eventually disappeared (Fig. 3c, Supplementary video S11). These results indicate that SLSs do not constitute a static, independent cluster of cells but are dynamically stabilized structures that continuously incorporate cells in front and leave cells trailing behind.

Collision of two independent SLSs. The SLS structure is stable regardless of collision other SLSs (Fig. 1c, Supplementary video S4). In order to investigate cell behaviour in colliding SLSs, SLS collisions were recorded at high magnification. In head-on collisions, the SLSs appeared to coalesce as clusters and glide in a circular motion (Supplementary video S11). They did not always

Table I | cAMP concentration in the extracellular fraction of $\mathrm{KI}-5$ cells with $5 \mathrm{mM}$ caffeine and conditioned medium

\begin{tabular}{lc} 
Addition & $\begin{array}{c}\text { cAMP concentration in } \\
\text { supernatant }(\mathrm{pmol} / \mathrm{mL})\end{array}$ \\
\hline $\begin{array}{l}\text { No caffeine and no CM } \\
5 \mathrm{mM} \text { caffeine and CM }\end{array}$ & $\begin{array}{l}1.70 \pm 0.33 \\
\text { The results are shown as the mean } \pm \text { S.D. of triplicate determinations of } 3 \text { independent } \\
\text { experiments. } C M=\text { conditioned medium (see Materials and methods). }\end{array}$ \\
\hline
\end{tabular}

a
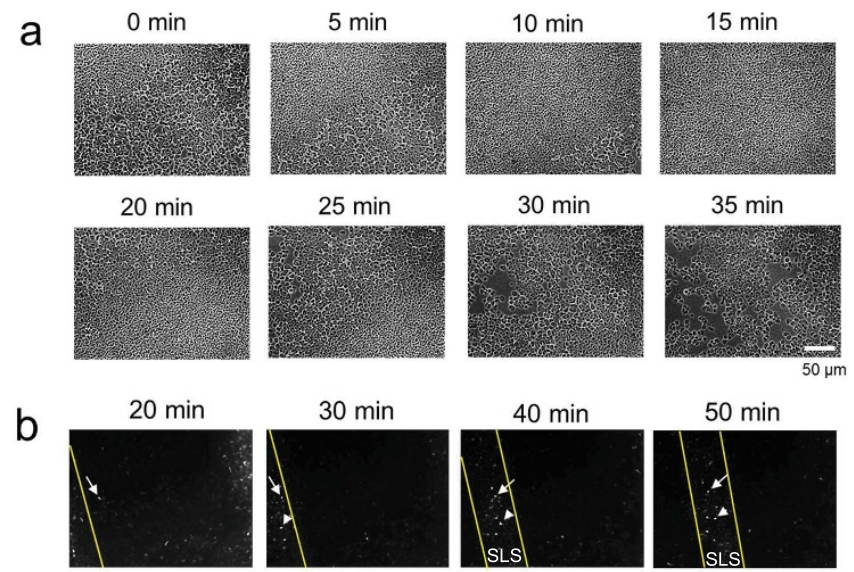

$30 \mathrm{~min}$

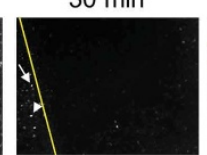

$40 \mathrm{~min}$

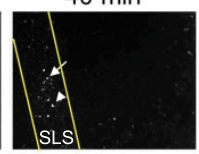

$50 \mathrm{~min}$

$60 \mathrm{~min}$

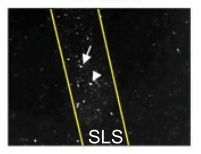

$70 \mathrm{~min}$

$80 \mathrm{~min}$
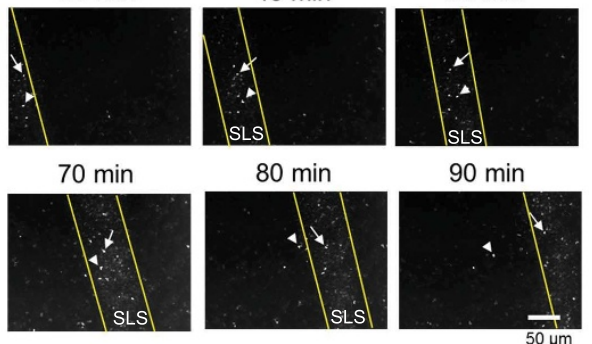

$90 \mathrm{~min}$

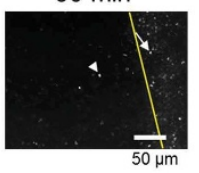

C
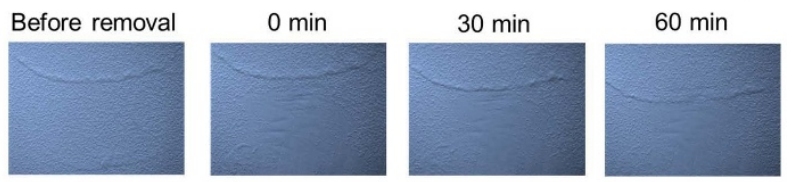

$90 \mathrm{~min}$

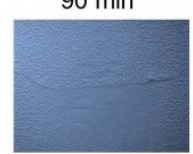

$120 \mathrm{~min}$

$150 \mathrm{~min}$
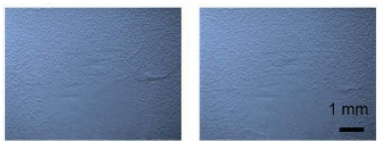

Figure $3 \mid$ Cell movements in SLSs. (a), Cell movement of SLSs. (b), Cell movement of SLSs with RFP-labelled KI-5 cells. (c), Removal of cells in front of a moving SLS. The arrow and arrowhead indicate cells incorporated into and left behind an SLS, respectively.

maintain their original orientation, with some switching orientation to the opposite direction. This was confirmed in RFPexpressing KI-5 cells (Fig. 4, Supplementary video 12, 13). Some cells in each soliton maintained (arrow in Fig. 4) or reversed (arrowhead in Fig. 4) direction after the collision. These results indicate that, in SLS collisions, cells merge into each other as a cluster and glide, but separate in 2 directions independent of the original direction.

End-to-end adhesion may play a major role in the distinct and stable directional locomotion of SLS. Next, cells comprising SLS were moderately dispersed and placed under an agar overlay (Fig. 5a, Supplementary video 14). A population of cells contacted each other in an end-to-end manner and moved in a tandem structure, similar to cell movement in slugs ${ }^{23}$. Within this structure, two or more cells were often found to concatenate although they sometimes formed a broken line (arrows in Fig. 5a). These end-to-end concatenations and

Table II | The number of highly REP-expressing cells that are incorporated into and left behind a SLS

\begin{tabular}{lccc} 
& \multicolumn{3}{c}{ The number of RFP-expressing } \\
\cline { 2 - 4 } & incorporated cells & cells behind & ratio \\
\hline Experiment I & 17 & 17 & 1.0 \\
Experiment II & 17 & 20 & 0.8 \\
Experiment III & 18 & 20 & 0.9 \\
\hline
\end{tabular}

Ratio indicates the relative value of the number of RFP-expressing incorporated cells to cells behind a SLS per movie. 
0 min
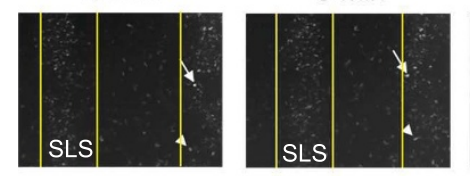

$25 \min$

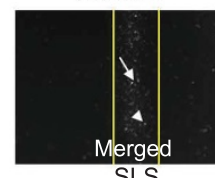

SLS
$10 \mathrm{~min}$

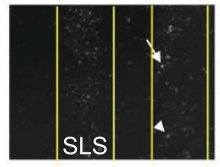

$30 \mathrm{~min}$

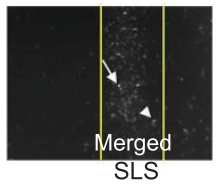

$15 \mathrm{~min}$

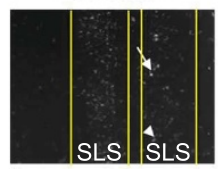

$35 \mathrm{~min}$

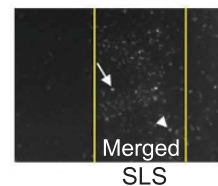

20 min

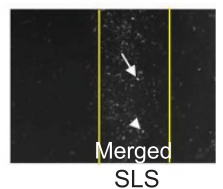

$40 \mathrm{~min}$

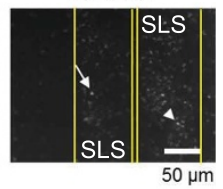

Figure 4 Collision of two independent SLSs with RFP-expressing KI-5 cells. The arrow and arrowhead indicate cells which maintained and changed direction, respectively, in SLSs after collision.

subsequent tandem structure movements were observed on occasions when one cell encountered other cells (arrowheads in Fig. 5a). Furthermore, SLS formation was never inhibited by $5 \mathrm{mM}$ EDTA at which concentration calcium-dependent cell adhesion is abolished and, thus, cell aggregation in wild-type cells is blocked by inhibition of side-by-side cell adhesion ${ }^{24}$ (Fig. 5b). These observations suggest end-to-end adhesion may play a major role in the distinct and stable directional locomotion of SLS, and calciumdependent cell adhesion is not involved in SLS formation.

\section{Discussion}

In this manuscript, we show that the distinct characteristics of solitons are observable in the mass cell movement of non-chemotactic mutants of a cellular slime mould. Upon starvation, wild-type cells of the cellular slime mould $D$. discoideum stop growing and initiate spontaneous formation of multicellular structures, accompanied by chemotaxis-dependent cell aggregation. This results in cell differentiation. In contrast, non-chemotactic mutants do not undergo aggregation; however, they do undergo a mass cell movement that possesses the characteristics of a soliton. They form pulsatile SLSs that retain their shape and move at a constant velocity. Furthermore, the shape of each SLS remains unchanged after collisions with other SLSs (Supplementary video S4, S12, S13). These observations provide the first insight into soliton-related behaviours in eukaryotic multicellular movement.

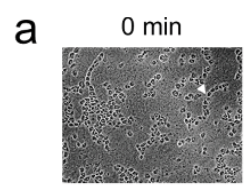

$4 \mathrm{~min}$

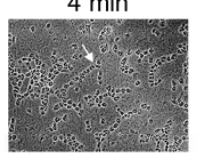

b

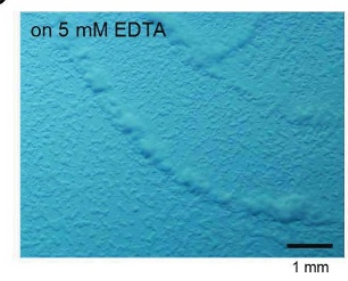

Figure $5 \mid$ Movement of the dispersed cells derived from SLSs. (a), Cell movement of loosely disaggregated SLS. Arrows indicate breakage of a cell concatenate. Arrowheads indicate attachment of 2 cell concatenates. (b), SLS formation on an agar plate containing 5 mM EDTA.
In myxobacteria, starved cells exhibit a highly organized periodic pattern of accumulations that move as traveling waves called ripples $^{25,26}$. Two sets of waves appear to move in opposite directions with the same wave length and speed, like SLSs; however, the mechanism of wave movement in collisions is rather different from that of SLS. When two ripple wave fronts collide, cells move in the reverse direction. Consequently, the outgoing waves consist of a combination of individuals from both incoming waves. This gives the appearance of waves passing through one another. In the collision of SLSs, each cell maintains its direction forward or reverses. This feature is distinctly different from that of SLS.

Null mutants that lack carA, gpaB, and $a c a A$ are defective in SLS formation. These observations indicate that cAMP signalling is responsible for SLS formation. On the other hand, extracellular cAMP and exhaustion of cAMP by caffeine had no effect on SLS formation. Thus, cAMP signalling is important for the transition from vegetative to starved stage, but is not necessary for SLS maintenance. Furthermore, since KI-5 is defective in intracellular cGMP metabolism in response to cAMP stimuli, cGMP is not necessary for SLS emergence and maintenance.

In $D$. discoideum, cell adhesion molecules mediate cell-to-cell contact during development. DdCAD-1 and gp80 are responsible for spatio-temporal cell adhesion. DdCAD-1 localizes primarily on cells at the tip and outer margin of cell streams, whereas gp80 localizes to contact regions between cells within well-developed streams ${ }^{27,28}$. DdCAD-1 is enriched in filopodial structures at the cell contact region, suggesting DdCAD-1 plays an important role at the leading edge of migrating cells. However, since we found that SLS formation and maintenance are EDTA-resistant phenomena, the adhesion molecule is not likely to be responsible for SLS, because DdCAD-1 is not adhesive under calcium-depleted conditions in the presence of EDTA. In the end-to-end adhesion in SLS, calcium-resistant adhesion molecules may serve as a target marker for contact. Based on these observations, the explanation for dynamically stable movement of SLS may be elucidated through the specific localization of calciumresistant adhesive molecules such as gp80.

Based on our observations, we speculate that the formation of SLSs is initiated by random collisions in early-stage starvation. Since SLS is only observed under starvation conditions, we suggest cell polarization is important for SLS. Once cells become polarized, their movement becomes aligned and propagates as a cluster by end-to-end adhesion. To understand if the characteristics of biological solitons reported in this study can apply to multicellular movement in not only $D$. discoideum but also general developmental morphogenesis, further mathematical treatment providing theoretical validation of the soliton nature of the SLSs is required.

\section{Methods}

Culture conditions and SLS observation. The parental strain of wild-type Dictyostelium discoideum XP55 and the non-chemotactic mutants KI-5 and KI-10 
cells were cultivated as described ${ }^{7}$. For each mutant, $1.0 \times 10^{5}$ cells were inoculated on a $9-\mathrm{cm} 1 / 3 \mathrm{SM}$ plate $(0.3 \%$ glucose, $0.3 \%$ bactopeptone, $1.5 \%$ agar, and $40 \mathrm{mM}$ $\mathrm{KH}_{2} \mathrm{PO} 4 / \mathrm{Na}_{2} \mathrm{HPO}_{4}, \mathrm{pH} 6.0$ ) with an aliquot of Klebsiella aerogenes suspension. At 2 days incubation at $21^{\circ} \mathrm{C}$, bacterial exhaustion was observed. Time-lapse videos were then taken at $21^{\circ} \mathrm{C}$ with a digital stereoscopic microscope (Olympus SZX12). Conditioned medium was prepared from the extracellular supernatant of wild-type cells $\left(1.0 \times 10^{7}\right.$ cells $\left./ \mathrm{mL}\right)$ incubated in phosphate buffer (PB: $10 \mathrm{mM} \mathrm{Na} / \mathrm{K}$ phosphate buffer, $\mathrm{pH}$ 6.5) for $12 \mathrm{~h}$ at $21^{\circ} \mathrm{C}$. To look at the effect of cell density on SLS, cells were cultivated on $1 / 3 \mathrm{SM}$ plates at $21^{\circ} \mathrm{C}$. They were harvested and washed 3 times with PB using a repeated centrifugation procedure $\left(350 \mathrm{~g}\right.$ for $2 \mathrm{~min} \times 3$ at $\left.4^{\circ} \mathrm{C}\right)$. The cells of each strain were plated with $1.5 \%$ non-nutrient agar and were adjusted to the cell density indicated in the figure legend. Higher-magnification time-lapse videos were taken using a modified agar overlay method as described ${ }^{29}$. Pictures and videos were taken using a contrast and fluorescence microscope (Zeiss Axiovert 200M).

Transformation of KI-5 cells. Transformation of KI-5 cells was performed as described previously with a few modifications ${ }^{30}$. Details are provided in the Supplementary methods.

1. Lakshmanan, M. Solitons, tsunamis and oceanographical applications of. in Extreme Environmental Events (ed Meyers, R. A.) 873-888 Springer, (2011).

2. Hasegawa, A. An historical review of application of optical solitons for high speed communications. Chaos 10, 475-485 (2000).

3. Heimburg, T. \& Jackson, A. D. On soliton propagation in biomembranes and nerves. Proc. Natl. Acad. Sci. USA 102, 9790-9795 (2005).

4. Sinkala, Z. Soliton/exciton transport in proteins. J. Theor. Biol. 241, 919-927 (2006)

5. Yakushevich, L. V. Is DNA a nonlinear dynamical system where solitary conformational waves are possible? J. Biosci. 26, 305-313 (2001).

6. Williams, J. G. Dictyostelium finds new roles to model. Genetics 185, 717-726 (2010).

7. Kuwayama, H., Ishida, S. \& Van Haastert, P. J. Non-chemotactic Dictyostelium discoideum mutants with altered cGMP signal transduction. J. Cell Biol. 123, 1453-1462 (1993).

8. Ohtsuka, H. Relationship between the movements of slug cells and chemotaxis in Dictyostelium discoideum. Master's Thesis, Kyoto University, Japan (in Japanese). (1994).

9. Pakes, N. K. et al. The Rac GEF ZizB regulates development, cell motility and cytokinesis in Dictyostelium. J. Cell Sci. 125, 2457-2465 (2012).

10. Sato, M. J. et al. Switching direction in electric-signal-induced cell migration by cyclic guanosine monophosphate and phosphatidylinositol signaling. Proc. Natl. Acad. Sci. USA 106, 6667-6672 (2009).

11. Siegert, F. \& Weijer, C. J. Three-dimensional scroll waves organize Dictyostelium slugs. Proc. Natl. Acad. Sci. USA 89, 6433-6437 (1992).

12. Swaney, K. F., Huang, C. H. \& Devreotes, P. N. Eukaryotic chemotaxis: a network of signaling pathways controls motility, directional sensing, and polarity. Аnnu. Rev. Biophys 39, 265-289 (2010).

13. Sun, T. J. \& Devreotes, P. N. Gene targeting of the aggregation stage cAMP receptor cAR1 in Dictyostelium. Genes Dev. 5, 572-582 (1991).

14. Kumagai, A. et al. Regulation and function of $\mathrm{G}$ alpha protein subunits in Dictyostelium. Cell 57, 265-275 (1989).

15. Pitt, G. S. et al. Structurally distinct and stage-specific adenylyl cyclase genes play different roles in Dictyostelium development. Cell 69, 305-315 (1992).

16. Kuwayama, H., Viel, G. T., Ishida, S. \& Van Haastert, P. J. Aberrant cGMPbinding activity in non-chemotactic Dictyostelium discoideum mutants. Biochim. Biophys. Acta 1268, 214-220 (1995).
17. Kuwayama, H. \& Van Haastert, P. J. Regulation of guanylyl cyclase by a cGMPbinding protein during chemotaxis in Dictyostelium discoideum. J. Biol. Chem. 271, 23718-23724 (1996)

18. Van Haastert, P. J., Jastorff, B., Pinas, J. E. \& Konijn, T. M. Analogs of cyclic AMP as chemoattractants and inhibitors of Dictyostelium chemotaxis. J. Bacteriol. 149, 99-105 (1982).

19. Alvarez-Curto, E., Weening, K. E. \& Schaap, P. Pharmacological profiling of the Dictyostelium adenylate cyclases ACA, ACB and ACG. Biochem. J. 401, 309-316 (2007).

20. Shapiro, R., Franke, J., Luna, E. J. \& Richard, H. Kessin. A comparison of the membrane-bound and extracellular cyclic AMP phosphodiesterases of Dictyostelium discoideum. Biochim. Biophys. Acta 758, 49-57 (1983).

21. Kay, R. R., Flatman, P. \& Thompson, C. R. DIF signalling and cell fate. Semin. Cell Dev. Biol. 10, 577-585 (1999).

22. Kuwayama, H. \& Kubohara, Y. Differentiation-inducing factor-1 and -2 function also as modulators for Dictyostelium chemotaxis. PLoS One 4, e6658 (2009).

23. Umeda, T. \& Inouye, K. Possible role of contact following in the generation of coherent motion of Dictyostelium cells. J. Theor. Biol. 219, 301-308 (2002).

24. Müller, K. \& Gerisch, G. A specific glycoprotein as the target site of adhesion blocking Fab in aggregating Dictyostelium cells. Nature 274, 445-459 (1978).

25. Welch, R. \& Kaiser, D. Cell behavior in traveling wave patterns of myxobacteria. Proc Natl. Acad. Sci. USA 98, 14907-10912 (2001).

26. Igoshin, O. A. \& Oster, G. Rippling of myxobacteria. Math. Biosci. 188, 221-233 (2004).

27. Sesaki, H. \& Siu, C. H. Novel redistribution of the $\mathrm{Ca}^{2+}$-dependent cell adhesion molecule DdCAD-1 during development of Dictyostelium discoideum. Dev. Biol. 177, 504-516 (1996)

28. Harloff, C., Gerisch, G. \& Noegel, A. A. Selective elimination of the contact site A protein of Dictyostelium discoideum by gene disruption. Genes Dev. 3, 2011-2019 (1989).

29. Fukui, Y., Yumura, S. \& Yumura, T. K. Agar-overlay immunofluorescence: high-resolution studies of cytoskeletal components and their changes during chemotaxis. Methods Cell Biol. 28, 347-356 (1987).

30. Kuwayama, H. \& Nagasaki, A. Desalted deep sea water increases transformation and homologous recombination efficiencies in Dictyostelium discoideum. J. Mol. Microbiol. Biotechnol. 14, 157-162 (2008).

\section{Acknowledgements}

We acknowledge Dr. K. Inouye for his support of and helpful discussion on this work.

\section{Author contributions}

H.K. performed all the experiments. H.K. and S.I. contributed equally to the discovery of the SLS. H.K. drafted and contributed to the final manuscript.

\section{Additional information}

Supplementary information accompanies this paper at http://www.nature.com/ scientificreports

Competing financial interests: The authors declare no competing financial interests.

How to cite this article: Kuwayama, H. \& Ishida, S. Biological soliton in multicellular movement. Sci. Rep. 3, 2272; DOI:10.1038/srep02272 (2013).

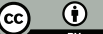

This work is licensed under a Creative Commons Attribution 3.0 Unported license. To view a copy of this license, visit http://creativecommons.org/licenses/by/3.0 